\title{
SUSY-GUTs, SUSY-Seesaw and the neutralino dark matter
}

\section{Lorenzo Calibbi}

Departament de Física Teòrica, Universitat de València-CSIC,

C/ Dr. Moliner 50, E-46100, Burjassot, Spain

E-mail: Iorenzo.calibbi@uv.es

\section{Yann Mambrini}

Laboratoire de Physique Théorique, Université Paris-Sud,

F-91405 Orsay, France

E-mail: yann.mambrini@th.u-psud.fr

\section{Sudhir Kumar Vempati}

Centre for High Energy Physics, Indian Institute of Science,

Bangalore 560 012, India

E-mail: vempati@cts.iisc.ernet.in

AbStRaCT: We will consider a SUSY-SU(5) with one right-handed neutrino with a large top like Yukawa coupling. Assuming universal soft masses at high scale we compute the low-energy spectrum and subsequently the neutralino LSP relic density taking also into consideration $\mathrm{SU}(5)$ as well as the see-saw running effects above the gauge coupling unification scale. We found that there exists no viable region in parameter space for $\tan \beta \lesssim 35$. The $\tilde{\tau}$ coannihilation process starts becoming efficient for $\tan \beta \gtrsim 35-40$. However, this process is significantly constrained by the limited range in which the stau is lighter than the neutralino. In fact, for a given $\tan \beta$ we find that there exists an upper bound on the lightest neutralino mass $\left(M_{\chi_{1}^{0}}\right)$ in this region. The A-pole funnel region appears at very large $\tan \beta \simeq 45-50$, while the focus-point region does not make an appearance till large $\left(m_{0}, M_{1 / 2}\right)$, namely a few $\mathrm{TeV}$. Large $A_{0}$ terms at high scale can lead to extended regions consistent with WMAP constraints and remove the upper bounds in the stau coannihilation regions.

KeYwords: GUT, Supersymmetry Phenomenology. 


\section{Contents}

1. Introduction 1

2. $\mathrm{SU}(5)_{\mathrm{RN}}$ and neutralino $\mathrm{DM}$

3. Spectrum and constraints 5

4. $\mathrm{SU}(5)_{\mathrm{RN}}$ and $\Omega_{\mathrm{DM}}$

4.1 Coannihilation region 10

4.2 A-pole funnel 12

4.3 Focus point region 13

$4.4\left|A_{0}\right|=3 m_{0}$

5. Remarks 13

\section{Introduction}

The presence of a natural Dark Matter (DM) candidate has been one of the hallmarks of low energy supersymmetry models (SUSY). Within the Minimal Supersymmetric Standard Model (MSSM), imposition of R-parity conservation would lead the lightest SUSY particle (LSP) to be stable and preferably neutral and a color singlet, a perfect candidate to explain the Dark Matter (DM) relic density in terms of a WIMP (Weakly Interacting Massive Particle) [四. With the advent of precision cosmology, pinnacle of it being the recent results from the WMAP experiment [2], the dark matter relic density is now known with high accuracy [3]. Together with availability of state-of-the-art numerical tools it is now possible to compute the relic density within a given SUSY model accurately up to few percent level.

However, it has been known that within most SUSY models, the relic density computed is either too large or too small; it is required that certain precise or critical relations between the various soft SUSY breaking parameters to exist in order the neutralino relic density comes out consistent with the measurements. The accuracy with which these relations need to be satisfied typically leaves out very tiny special regions in the parameter space where neutralino relic density is satisfactory; the tiny regions can be traced back to existence of one of the relations between the parameters 4 .

In the simplest models of gravity mediation, i.e, minimal supergravity (mSUGRA), which will be the focus of our work, the soft terms have 'universal' boundary conditions at a high energy scale, such as the Grand Unification scale, $M_{\mathrm{GUT}}$. The lightest supersymmetric particle (LSP) is the lightest neutralino $\left(\tilde{\chi}_{1}^{0}\right)$ in most of the parameter space. There exists 
a)

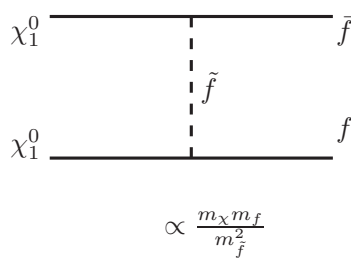

b)

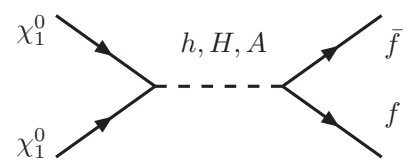

$\propto \frac{m_{x}^{2}}{M_{A}^{2}} \frac{Z_{11} Z_{13,14}}{m_{W}} m_{f}(\tan \beta)^{-2 I_{3}^{f}}$

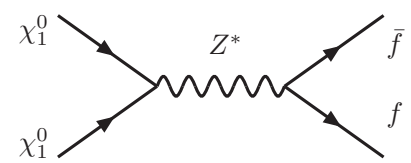

$\propto \frac{m_{f} m_{X}}{m_{Z}^{2}}\left(-\left|Z_{13}\right|^{2}+\left|Z_{14}\right|^{2}\right)$
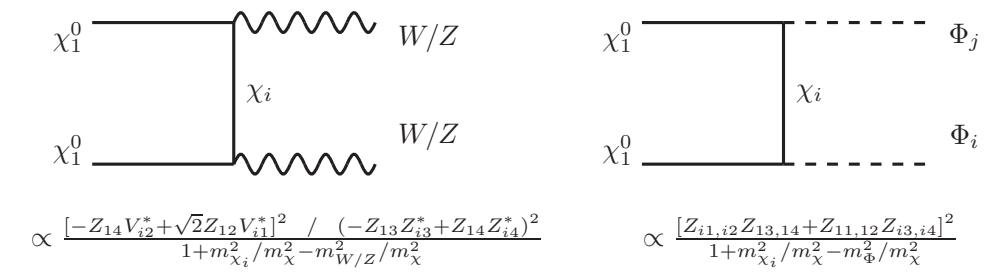

Figure 1: Feynman diagrams for LSP neutralino annihilation into a fermion pair (a) and into massive gauge bosons and Higgs bosons (b). The relevant parts of the amplitudes are shown explicitly. $V$ and $Z$ are the chargino and neutralino mixing matrices.

three regions where viable DM relic density is possible after taking into account the existing low energy direct and indirect constraints on the supersymmetric spectrum including the recent constraints from LEP measurements [5]. These are (i) The stau coannihilation region, (ii) The A-pole funnel region and (iii) The focus-point or the hyperbolic branch region. In the region (i), where the lightest stau ( $\left.\tilde{\tau}_{1}\right)$ mass is very close to the LSP mass [6], significant enhancement in the annihilation cross-section happens due to the coannihilation between the lightest neutralino and the $\tilde{\tau}_{1}[0,8]$ (ii) In the so-called funnel region, resonant enhancement of the annihilation cross-section takes place in the process $\left(\tilde{\chi}_{1}^{0} \tilde{\chi}_{1}^{0} \rightarrow f \bar{f}\right)$ through the intermediate state of $(h, A, H)$, where $h, H, A$ represent light neutral, heavy neutral and the pseudo-scalar higgs respectively [9]. And finally the focus-point region (iii) is the region with large universal soft parameters which however leads to a small weakscale value of $\mu$ (the Higgs bilinear coupling in the superpotential), raising the Higgsino component in the LSP, and thus leading to significant enhancement in the annihilation cross-section [10].

A crucial aspect of this arrangement of things where SUSY DM would require some critical relations to be satisfied between various parameters is that these relations would not be stable under slightest modifications to the SUSY soft masses. These modifications are natural when we consider extensions of the minimal supersymmetric standard model (MSSM), where MSSM is embedded into either a SUSY Grand Unified Theory (SUSY-GUT) and/or augmented with a see-saw mechanism. The additional radiative effects present in these models are sufficient to significantly modify the spectrum at the weak scale. More importantly, these effects can destabilize the special regions in the parameter space where these relations hold or sometimes these modifications can be so large that there might be no parameter space left where these special relations can hold. This is precisely the issue we wish to address in our present work.

We will consider a SUSY-SU(5) with one right-handed $(\mathrm{RH})$ neutrino $\left(\mathrm{SU}(5)_{\mathrm{RN}}\right)$ with 
a large top like Yukawa coupling. ${ }^{1}$ Such a model is motivated from the requirement of non-zero neutrino masses and can have UV completion in $\mathrm{SO}(10)$ based models. Assuming universal soft masses at a scale $M_{X} \simeq 10^{17} \mathrm{GeV}$, we compute the low-energy spectrum taking also into consideration $\mathrm{SU}(5)$ as well as the see-saw running effects above the gauge coupling unification scale $\left(M_{\mathrm{GUT}} \simeq 10^{16} \mathrm{GeV}\right)$. Adding the effects of the further running, down to the weak scale, we compute the relic density with the weak scale spectrum.

Firstly, we found that there exists no viable region in parameter space for $\tan \beta \lesssim 35$ for neutralino dark matter. Parameter space points consistent with WMAP results start appearing with $\tan \beta \gtrsim 35$ and increase with increasing $\tan \beta$. However, this process is highly constrained by the limited range in which the stau is lighter than the neutralino. In fact, we find that the coannihilation region is significantly modified with respect to the MSSM and it has the shape of a trunk, in the $\left(m_{0}, M_{1 / 2}\right)$ plane, ${ }^{2}$ cutting into the $M_{1 / 2}$ axis. Thus, for a given $\tan \beta$, there exists an upper bound on the lightest neutralino mass $\left(M_{\chi_{1}^{0}}\right)$. The allowed parameter space widens up at very large $\tan \beta \simeq 50$ where the A-pole funnel region appears. The focus-point region is very difficult to realise in this model because electroweak symmetry breaking is much easier to achieve compared to the standard MSSM. In fact, even going up to $5 \mathrm{TeV}$ in $\left(m_{0}, M_{1 / 2}\right)$ plane, focus-point region does not make an appearance.

While the above results are particularly true as long as the universal trilinear coupling $A_{0}=0$ at the high scale, the results can significantly change with large $-3 m_{0} \lesssim A_{0} \lesssim$ $3 m_{0}$ boundary conditions which due to the possibility that some of the masses becoming tachyonic, significantly affects the viable parameter space. For instance, for $A_{0}=+3 m_{0}$, the stau coannihilation region branches out into two regions, a new region other than the trunk region discussed above. The A-pole funnel also spreads giving rise to a larger region of the parameter space which is viable under WMAP constraints.

The rest of the paper is organized as follows: in the next section, we will elaborate our framework along with revising some aspects of neutralino dark matter. In section 3 , we summarize the constraints we use in our numerical analysis as well as sketch our computing procedure. In section 4 , we discuss our results and close with a discussion in our final section.

\section{2. $\mathrm{SU}(5)_{\mathrm{RN}}$ and neutralino $\mathrm{DM}$}

As mentioned in the introduction, in the following we will consider a simple SUSY-GUT framework based on SU(5). Further we also add a RH neutrino with a large top-like Yukawa coupling, which is natural when this model is incorporated in an $\mathrm{SO}(10)$ model [11]. The superpotential of this framework is given by:

$$
W_{\mathrm{SU}(5)_{\mathrm{RN}}}=\frac{1}{2} h_{i i}^{u} 10_{i} 10_{i} 5_{u}+h_{3}^{\nu} \overline{5}_{3} 15_{u}+h_{i j}^{d} 10_{i} \overline{5}_{j} \overline{5}_{d}+\frac{1}{2} M_{R} 11,
$$

\footnotetext{
${ }^{1}$ We need at least one more RH neutrino and a corresponding Yukawa coupling to fit the neutrino data. This Yukawa coupling could be much smaller than the top Yukawa and thus its effects negligible to the discussion pertaining here.

${ }^{2} m_{0}$ and $M_{1 / 2}$ are the universal mSUGRA boundary conditions for the scalars and gauginos masses, respectively.
} 
$i, j$ being generation indices and 10 and $5(\overline{5})$, representing the tensorial and fundamental (anti-fundamental) representations of $\mathrm{SU}(5)$ respectively. The subscript $u, d$ denote the up and down type Higgs fields. ${ }^{3}$ Two comments are in order before we proceed further.

Firstly, to accommodate neutrino masses, we would need at least one more RH neutrino in addition to the one already present in eq. (2.1). However, in most models, the corresponding Yukawa couplings are not as large as the top Yukawa coupling. ${ }^{4}$ Thus, the impact of the second and third neutrino Yukawa couplings even if they are present is expected to be minimal on the discussion below. In fact, we have explicitly checked this to be the case in models where the neutrino Yukawa coincides with the up-type Yukawa matrix. Secondly, keeping $\mathrm{SO}(10)$ completion in mind, we have chosen the high scale $M_{X}$ to be around $\simeq 10^{17} \mathrm{GeV}$. Another natural choice would be the reduced Planck scale, where $M_{X} \simeq 10^{18} \mathrm{GeV}$. Nevertheless, with this choice of the scale, we believe our limits on $\tan \beta$ (as well as the neutralino mass) will only become stronger. In section 1 we will briefly comment about the dependence of our results on the choice of $M_{X}$. For most of the discussion, however, we will just choose the lower value for $M_{X}$.

The soft SUSY-breaking terms are considered to be universal at the scale, $M_{X}$. These contain the standard mass-squared terms for the scalar particles (the squarks and the sleptons), the bilinear Higgs scalar couplings, the trilinear scalar couplings as well as the mass terms for the gauginos. Given that R-parity is conserved, the LSP remains stable. In most models of SUSY-breaking like mSUGRA, the LSP is the lightest neutralino. The neutralinos are the physical superpositions of the fermionic partners of the neutral electroweak gauge bosons, called bino $\left(\tilde{B}^{0}\right)$ and wino $\left(\tilde{W}_{3}^{0}\right)$, and of the fermionic partners of the neutral Higgs bosons, called Higgsinos $\left(\tilde{H}_{u}^{0}, \tilde{H}_{d}^{0}\right)$. We can express the lightest neutralino as

$$
\tilde{\chi}_{1}^{0}=Z_{11} \tilde{B}+Z_{12} \tilde{W}_{3}+Z_{13} \tilde{H}_{d}^{0}+Z_{14} \tilde{H}_{u}^{0}
$$

where $Z_{i j}$ are the elements of the neutralino mixing matrix. It is commonly defined that $\tilde{\chi}_{1}^{0}$ is mostly gaugino-like if $P \equiv\left|Z_{11}\right|^{2}+\left|Z_{12}\right|^{2}>0.9$, Higgsino-like if $P<0.1$, and mixed otherwise. This factor $P$ has a major impact in determining the relic abundance of the LSP as it determines the rate of neutralino annihilation cross-section. In figure 1 we show the relevant Feynman diagrams contributing to neutralino annihilation. This can be computed for a given SUSY spectrum, which depends on the SUSY breaking model.

In the MSSM, with universal soft terms at $M_{\mathrm{GUT}}=2 \times 10^{16} \mathrm{GeV}$ (CMSSM), for most of the parameter space, the lightest neutralino is mainly bino and, as a consequence, the annihilation cross-section is small producing too large relic abundance. Nevertheless, as mentioned in the introduction, three corridors exist where the cross-section is enhanced and the WMAP bounds can be satisfied. First, there is the coannihilation branch, i.e. the region where the stau mass is almost degenerate with that of LSP. Another corridor corresponds to the A-pole region. This occurs in the parameter space where $4\left(m_{\tilde{\chi}_{1}^{0}}\right)^{2} \simeq$ $m_{A}^{2} \simeq m_{H_{1}}^{2}-m_{H_{2}}^{2}-M_{Z}^{2}$, with $m_{H_{i}}^{2}$ being the soft Higgs mass terms, and the dominant neutralino annihilation process is through the 'resonant' s-channel pseudo-scalar Higgs

\footnotetext{
${ }^{3}$ We have written the superpotential in the basis where up-type quarks are diagonal.

${ }^{4}$ In fact, if one considers models based on $\mathrm{SO}(10)$, it can be as small as the charm Yukawa, $y_{c}$.
} 


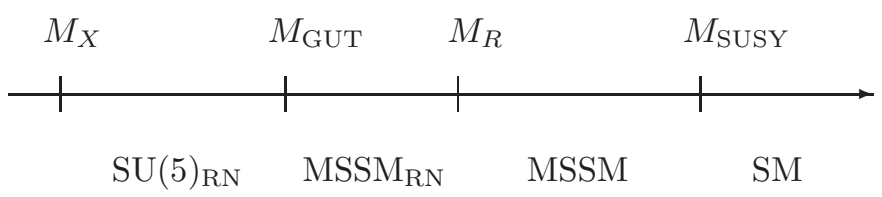

Figure 2: Schematic picture of the energy scales involved in the model.

exchange. Finally, there is a 'Higgsino' corridor close to the no electroweak symmetry breaking (No EWSB) region. In this region the $\mu$ parameter is much smaller than the bino mass and the lightest neutralino has a larger Higgsino component (thus annihilating efficiently via $Z$ boson exchange).

As we have stressed in the introduction, these corridors or special regions in the parameter space are not stable under modifications to the soft spectrum. In this connection, it was already noted, for e.g. in refs. [12], that the annihilation cross-section can change significantly if the soft terms determined at the unification scale, $M_{\mathrm{GUT}}$, are non-universal. Similarly, ref. [13] assumed no-scale boundary conditions at $M_{X}=M_{\text {Planck }}$, which would result in non-universal boundary conditions at $M_{\mathrm{GUT}} \cdot{ }^{5}$ In the scheme we are considering, which has been elaborated in the beginning of this section, something similar happens: the soft terms are indeed non-universal at the GUT scale and, as we will discuss in detail below, this would have implications for the regions in the parameter space where viable relic neutralino density was possible.

\section{Spectrum and constraints}

Before proceeding further in the present section, we will provide details of our computation of the supersymmetric spectrum in the framework we have considered and the various constraints we have used. A schematic diagram describing the integration procedure is presented in figure 2. As inputs at the weak scale, we consider the Yukawa couplings of the up-type quarks, down-type quarks, charged leptons and $\tan \beta$. We consider one generation of neutrino with a mass around $0.05 \mathrm{eV}$. We use 1-loop RGEs to run all the Yukawa couplings up to the high scale. A more detailed description of the procedure can be found in ref. 14]. After several iterations to check consistencies between neutrino-scale spectrum and $M_{X}$, we are now ready to compute the spectrum from the high scale to the weak scale. The soft parameters, gauge and Yukawa couplings follow the SU(5) RN RGEs 15] between $M_{X}$ and $M_{\mathrm{GUT}}$. We then compute the evolution of these SUSY parameters using a modified version of the Fortran package SUSPECT [16, 17] where we have included one $\mathrm{RH}$ sneutrino between the GUT scale and the RH neutrino mass scale, $M_{R}$. The runnings then follows the classical MSSM RGEs after $M_{R}$ where the RH neutrino sector decouples. The low energy mass spectrum is then calculated using SUSPECT. The evaluation of the $b \rightarrow s \gamma$ branching ratio, the anomalous moment of the muon and the relic neutralino den-

\footnotetext{
${ }^{5}$ Our work is closest in spirit to this work and could be considered a generalization of it.
} 
sity is carried out using the routines provided by the program micrOMEGAs1.3.1 [18]. The following are the list of constraints we apply on the soft spectrum:

1. Electroweak symmetry breaking.

Minimizing the Higgs potential in the MSSM leads to the standard (tree-level) relation

$$
\mu^{2}=\frac{-m_{H_{2}}^{2} \tan ^{2} \beta+m_{H_{1}}^{2}}{\tan ^{2} \beta-1}-\frac{1}{2} M_{Z}^{2},
$$

This minimization condition is imposed at the scale $M_{\mathrm{SUSY}} \equiv \sqrt{m_{\tilde{t}_{1}} m_{\tilde{t}_{2}}}$, where $m_{\tilde{t}_{i}}$ are the stop masses. eq. (3.1) can be approximated in most cases by

$$
\mu^{2} \simeq-m_{H_{2}}^{2}-\frac{1}{2} M_{Z}^{2}
$$

When the right-hand side is negative, electroweak breaking cannot occur. The Higgs mass parameter $m_{H_{2}}^{2}$ is positive at the GUT scale, but decreases with decreasing scale down to $M_{\mathrm{SUSY}}$, through the contributions it receives from RG running at the scale $\tilde{\mu}$; in presence of $\mathrm{RH}$ neutrinos, the $\mathrm{RG}$ equation for $m_{\mathrm{H}_{2}}^{2}$ reads:

$$
(4 \pi)^{2} \frac{\partial m_{H_{2}}^{2}}{\partial \ln \left(\tilde{\mu} / M_{X}\right)} \simeq 6 y_{t}^{2}\left(m_{H_{2}}^{2}+m_{\tilde{U}_{3}}^{2}+m_{\tilde{Q}_{3}}^{2}+A_{t}^{2}\right)+2 y_{\nu}^{2}\left(m_{H_{2}}^{2}+m_{\tilde{N}}^{2}+m_{\tilde{L}_{3}}^{2}+A_{\nu}^{2}\right)
$$

where gauge contributions have been neglected. Here $m_{\tilde{Q}_{3}}^{2}$ and $m_{\tilde{U}_{3}}^{2}$ corresponds to the $\tilde{t}_{L}$ and $\tilde{t}_{R}$ soft masses, while $m_{\tilde{L}_{3}}^{2}, m_{\tilde{N}}^{2}$ are the same for the left-handed and right-handed sneutrinos respectively; $y_{t}$ and $y_{\nu}$ are the top and the neutrino Yukawa couplings, while $A_{t}$ and $A_{\nu}$ the corresponding soft SUSY-breaking trilinear couplings. Typically, in the MSSM the value of $m_{H_{2}}^{2}$ at the scale $M_{\mathrm{SUSY}}$ depends mainly on the gluino mass $M_{3}$ at the GUT scale (via its effect of increasing $m_{\tilde{U}_{3}}^{2}$ and $m_{\tilde{Q}_{3}}^{2}$ ). However, in our case, the further increasing of $m_{\tilde{U}_{3}}^{2}$ and $m_{\tilde{Q}_{3}}^{2}$ due to the GUT running between $M_{X}$ and $M_{\mathrm{GUT}}$, together with the positive additional contribution proportional to $y_{\nu}^{2}$, drives $m_{H_{2}}^{2}$ toward even more negative values. As a consequence, the parameter space region where $\mu^{2}<0$ (or No EWSB region) is much more reduced, and even absent in our case as we will see in the analysis.

\section{The mass spectrum constraints.}

We have implemented in our analysis the lower bounds on the masses of SUSY particles and of the lightest Higgs boson. In the squark and slepton sector we checked for the occurrence of tachyons. We applied in our analysis the LEP2 lower bound limit on the mass of the lightest chargino $m_{\tilde{\chi}_{1}^{+}}>103.5 \mathrm{GeV}$. In the non-tachyonic region, typically, the most constraining is the lightest Higgs boson mass constraint. In the decoupling limit $\left(M_{A} \gg M_{Z}\right.$, applicable in all our parameter space), $m_{h}>114 \mathrm{GeV}$ at $3 \sigma$. This bound is very sensitive to the value of the top mass. We have taken $m_{t}=174 \mathrm{GeV}$ throughout our analysis.

3. The $b \rightarrow s \gamma$ branching ratio. 
One observable where SUSY particle contributions might be large is the radiative flavor changing decay $b \rightarrow s \gamma$. In the Standard Model this decay is mediated by loops containing the charge $2 / 3$ quarks and $W$-bosons. In SUSY theories additional contributions come from loops involving charginos and stops, top quarks and charged higgs bosons or, in presence of RG induced mixing in the down squarks sector, down squarks and gluinos. The measurements of the inclusive decay $B \rightarrow X_{s} \gamma$ at CLEO 19] and BELLE [20], leads to restrictive bounds on the branching ratio $b \rightarrow s \gamma$. We impose in our analysis $2.33 \times 10^{-4} \leq B R(b \rightarrow s \gamma) \leq 4.15 \times 10^{-4}$ at the $3 \sigma$ level. We mostly choose $\mu>0$ enabling cancellations between chargino contributions and charged Higgs contributions 21.

Before proceeding further, we elaborate a bit more about the flavour processes in our model. Since we are in a Grand Unified theory, one would expect large off-diagonal entries to be generated in the soft matrices at the GUT scale due to RG running effects from $M_{X}$ to $M_{\mathrm{GUT}}$. This could have large effects in flavour processes at weak scale. For example, a large off-diagonal entry in the 23 sector of the down squarks would contribute dominantly through the gluino interactions to the $b \rightarrow s+\gamma$ rate leading to significant constraints on that entry, unless of course, there are accidental destructive interferences among the various contributions already mentioned above. However, in our case, even if the corresponding mass matrices are non-universal at the GUT scale, the entries are generically small as they are proportional to the CKM entries. Some ambiguity can arise in the way we handled the neutrino sector. If all the three right handed neutrinos are present and the neutrino Dirac Yukawa matrix carries large "left"-mixing then they can generate large off-diagonal entries at the $M_{R}$ scale. In such a case a combination of both leptonic and hadronic flavour violating constraints have to be used [22]. On the other hand, it is known that even small departures from universality at high energy may have a strong effect on $b \rightarrow s+\gamma$, especially tending to make the constraint weaker [23]. In the present case, the constraint will be a bit weaker because $\mathrm{RH}$ neutrinos in $\mathrm{SU}(5)$ only affect the down squarks 'RR' sector. Moreover, we can avoid these effects at least in our framework by choosing small left mixing, though flavour violation in the leptonic sector might still be significant even in that case [24, 14]. We postpone such an analysis for future.

\section{The anomalous moment of the muon.}

We have also taken into account the SUSY contributions to the anomalous magnetic moment of the muon, $a_{\mu}=\left(g_{\mu}-2\right) / 2$. We used in our analysis the recent experimental results for the muon anomalous magnetic moment [25], as well as the most recent theoretical evaluations of the Standard Model contributions [26]. It is found that when $e^{+} e^{-}$data are used the experimental excess in $\left(g_{\mu}-2\right)$ would constrain a possible SUSY contribution to be $7.1 \times 10^{-10} \lesssim a_{\mu}^{\mathrm{SUSY}} \lesssim 47.1 \times 10^{-10}$ at $2 \sigma$ level. However when tau data is used a smaller discrepancy with the experimental measurement is found. In order not to exclude the latter possibility, when analyzing the parameter space with $\mu>0$ we will simply plot contours with the relevant value $a_{\mu}^{\mathrm{SUSY}}=7.1 \times 10^{-10}$. 


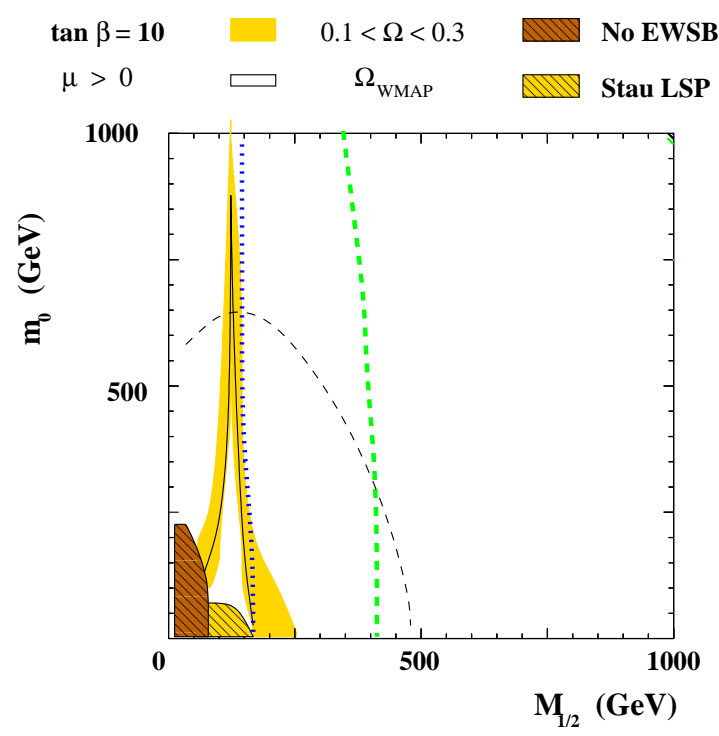

Figure 3: The $\left(m_{0}, M_{1 / 2}\right)$ plane with all the low-energy constraints on the parameter space. While the color code is explained above, the green dashed line indicates the Higgs mass bound from the LEP, while the dark dashed line represents the $b \rightarrow s+\gamma$ limit.

\section{Relic density.}

Our basic assumption is that the LSP is stable on cosmological time scales. Furthermore we will assume that the LSP abundance is thermal. Within such framework the regions of the parameter space that lead to overproduction of dark matter are excluded. On the other hand, the regions that yield LSP abundance below the WMAP limit are not considered as excluded (though as less favored), but simply require non-thermal production or a dark matter candidate beyond the soft spectrum. The WMAP collaboration gives the $3 \sigma$ narrow limit [2]

$$
0.087 \lesssim \Omega_{\mathrm{DM}} h^{2} \lesssim 0.138
$$

on the dark matter relic abundance.

\section{4. $\mathrm{SU}(5)_{\mathrm{RN}}$ and $\Omega_{\mathrm{DM}}$}

Before going to present the numerical results of our analysis, let us briefly mention the major changes to the spectrum one could expect in our framework and the possible implications on the neutralino relic density. As we have mentioned in the previous section, we have assumed that the soft masses are universal at a scale $M_{X}$ which we have chosen to be around $10^{17} \mathrm{GeV}$. As far as the neutralino relic density is concerned, two major effects on the soft spectrum due to the SU(5) running between the scale $M_{X}$ and $M_{\mathrm{GUT}}$ and subsequently to $M_{R}$ can be given as [14]:

- The right-handed slepton $\tilde{\tau}_{R}$ now sits in the 10 and thus it will receive contributions from the full gaugino multiplet of the SU(5). At the leading log level, these 
contributions are given at the GUT scale as:

$$
m_{\tilde{\tau}_{R}}^{2}\left(M_{\mathrm{GUT}}\right) \simeq \frac{144}{20 \pi} \alpha_{5} M_{1 / 2}^{2} \ln \left(\frac{M_{X}}{M_{\mathrm{GUT}}}\right) \simeq 0.25 M_{1 / 2}^{2}
$$

where we have taken the limit $m_{0} \rightarrow 0$ and $\alpha_{5}=\frac{g_{5}^{2}}{4 \pi}$ represents the unified gauge coupling at $M_{\mathrm{GUT}}$. This large positive contribution to the $\tilde{\tau}_{R}$ mass makes the stau heavier than the LSP (which is the lightest neutralino) for most of the parameter space. Let's note here that choosing a larger value for $M_{X}$ (such as $M_{\text {Planck }}$ ) would further increase $m_{\tilde{\tau}_{R}}^{2}$, strengthening the effect described above.

- In our framework the neutrino Yukawa coupling taking part in the see-saw mechanism as shown in eq. (2.1) is taken to be as large as the top Yukawa coupling. This introduces an additional top-Yukawa like coupling to the up-type Higgs from the scales $M_{X}$ down to $M_{R}$. Compared to the case of MSSM with RH neutrinos, as shown in eq. (3.3), here the length of running is more and thus more effective radiative electroweak symmetry breaking takes place. ${ }^{6}$ Moreover, the increasing of the upsquarks soft masses, due to the unified gauge sector, contributes to pushing $m_{H_{2}}^{2}$ down to more negative values than in the CMSSM. As a consequence, for most of the parameter space, we find that electroweak symmetry breaking is viable unlike in the CMSSM where substantial amount of parameter space is ruled out.

The above two reasons are sufficient to offset the conditions which give viable dark matter in CMSSM at low $\tan \beta$. As stated in the introduction, we found no viable region giving the required relic density up to $\tan \beta \lesssim 35$. In figure 3 , we show the non-existence of any region of the parameter space which gives rise to the correct relic density in our framework of $\mathrm{SU}(5)_{\mathrm{RN}}$, for $\tan \beta=10$ and $A_{0}=0$. All the three branches of CMSSM are not possible here ${ }^{7}$ as none of their corresponding relations could be satisfied within this regime.

To make this statement more concrete, in figure 1, we plot all the points which satisfy the available direct/indirect constraints and give viable relic density as a function of $\tan \beta$ and LSP mass. From the figure we see that viable DM is only possible for values of:

$$
\tan \beta \gtrsim 34 ; \quad m_{\tilde{\chi}_{1}^{0}} \gtrsim 150 \mathrm{GeV}
$$

These are quite strong lower bounds on the neutralino mass and $\tan \beta$ and will be useful in distinguishing this model compared to the standard CMSSM parameter space. In the figure 1 , we see that the small strip of points on the lower left corner gives a more constrained solution in $m_{\tilde{\chi}_{1}^{0}}$ for $\tan \beta \lesssim 45$. For larger $\tan \beta$, we see that almost all possible values are

\footnotetext{
${ }^{6}$ The value of $M_{R}$ is uniquely fixed $\left(M_{R} \approx 6 \times 10^{14} \mathrm{GeV}\right)$, through the see-saw mechanism, by our choice of the neutrino Yukawa coupling and light neutrino mass scale. Some variation in $M_{R}$ can come in a complete three generation model choosing inverse/normal hierarchy and particular mixing patterns. Choosing a larger light neutrino mass $(\mathcal{O}(\mathrm{eV}))$ would simply lower $M_{R}$, thus increasing the RG effect on $\mu$.

${ }^{7}$ Even if we have confined ourselves, in the figure, to the parameter space below $1 \mathrm{TeV}$ for both $m_{0}$ and $M_{1 / 2}$, we have performed a scanning up to $5 \mathrm{TeV}$, without finding viable regions.
} 


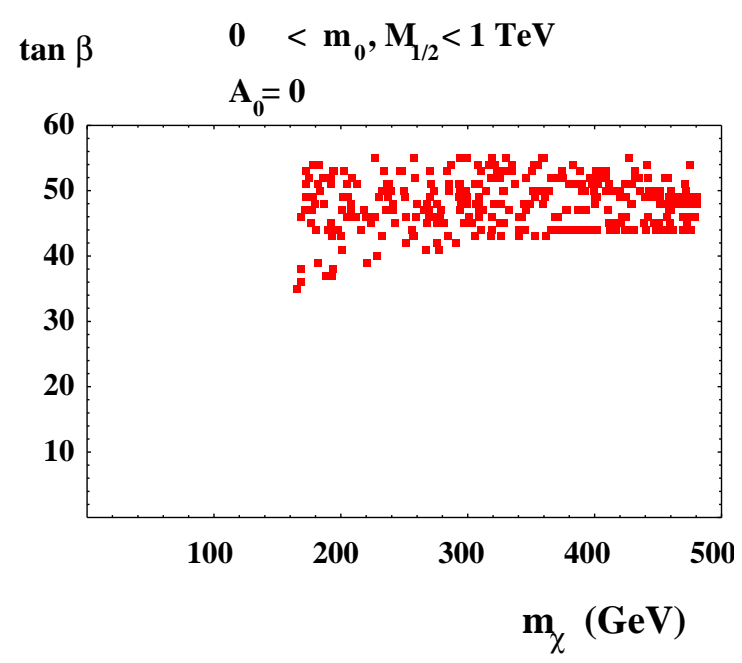

Figure 4: Points allowed by experimental and theoretical constrains after a scan on $(0<$ $\left.m_{0}, M_{1 / 2}<1 \mathrm{TeV}\right)$ and $(20<\tan \beta<55)$

allowed in the lightest neutralino mass. However, at this level, we are unable to distinguish between the actual annihilation processes involved in generating the correct relic density. A more detailed look shows us the existence of two regions which we elaborate here.

\subsection{Coannihilation region}

As we have discussed previously, the large pre-GUT scale contributions to the $\tilde{\tau}_{R}$ in eq. (4.1) make the lightest stau heavier than the LSP in most of the parameter space. However, when $\tan \beta$ becomes large, it is possible to make the lightest stau $\tilde{\tau}_{1}$ closer to the LSP mass by making the left-right (LR) mixing term $m_{\tau}\left(A_{\tau}-\mu \tan \beta\right)$ large. This is what indeed happens in our case. Stau coannihilation cross-sections start becoming effective for $\tan \beta \gtrsim 34$, leading to relic density within the WMAP bound. In figure 5 we have plotted, the WMAP compatible regions for the case of CMSSM as well as $\mathrm{SU}(5)_{\mathrm{RN}}$, for $\tan \beta=40$ and $A_{0}=0$.

Two features are evident from such a comparison between the CMSSM and the $\mathrm{SU}(5)_{\mathrm{RN}}$ : (i) the WMAP compatible region is much smaller in the case of $\mathrm{SU}(5)_{\mathrm{RN}}$ and (ii) the shape of the allowed region is quite different in the case of $\mathrm{SU}(5)_{\mathrm{RN}}$ (we will call it trunk region). In fact, the allowed region cuts-off for a value of $M_{1 / 2} \simeq 520 \mathrm{GeV}$ in the above plot. This would correspond to a LSP mass of around $240 \mathrm{GeV}$; an LSP of higher mass would not be able to give rise to the correct stau-neutralino coannihilation rate. This we would say corresponds to an upper bound on the LSP mass which could give viable stau coannihilation rate such that the relic density is within the WMAP bound. The presence of such upper bound in the LSP mass is not peculiar of the model we are considering here, since similar bounds are well-known also within CMSSM [7, 27]. However, the nature of these upper bounds is quite different in the two models. In the CMSSM, the reason is that the coannihilation cross-section decreases at larger SUSY masses [8] and the coannihilation rate becomes too low even if the condition $m_{\tilde{\tau}_{1}} \approx m_{\tilde{\chi}_{1}^{0}}$ is satisfied. On the other hand, in 

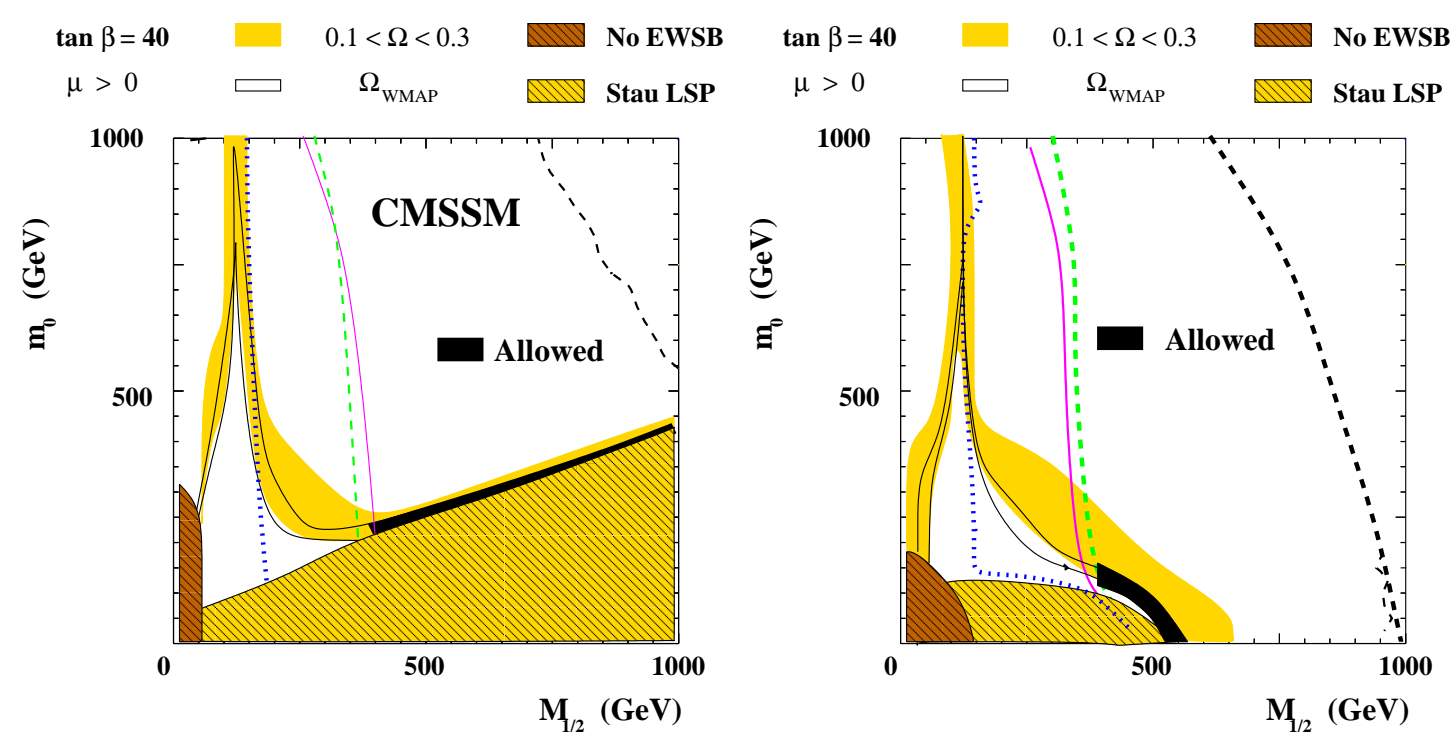

Figure 5: We show the coannihilation region for $\tan \beta=40$ for the case of CMSSM and SU $(5)_{\mathrm{RN}}$. The green dashed and the dark dashed lines represent same as above.

$\mathrm{SU}(5)_{\mathrm{RN}}$, the region for which $m_{\tilde{\tau}_{1}} \approx m_{\tilde{\chi}_{1}^{0}}$ simply disappears for some value of $M_{1 / 2}$, as a consequence of the peculiar shape of the $\tilde{\tau}$-LSP region. This excludes the possibility of efficient coannihilation even for values of $m_{0}$ and $M_{1 / 2}$ outside the " $1 \mathrm{TeV}$ box" of figure (as we numerically checked), so that the value $m_{\tilde{\chi}_{1}^{0}} \simeq 240 \mathrm{GeV}$ is a real upper bound for the case considered $\left(\tan \beta=40, A_{0}=0\right)$.

The reason for these peculiar features of $\mathrm{SU}(5)_{\mathrm{RN}}$ can again be traced back to the large gaugino contribution to the stau mass above the GUT scale. At the weak scale, roughly the stau mass matrix is now given by:

$$
\mathcal{M}_{\tilde{\tau}}^{2}=\left(\begin{array}{ll}
m_{\tilde{\tau}_{L L}}^{2} & m_{\tilde{\tau}_{L R}}^{2} \\
m_{\tilde{\tau}_{L R}}^{2} & m_{\tilde{\tau}_{R R}}^{2}
\end{array}\right),
$$

where, including the pre-GUT effects,

$$
m_{\tilde{\tau}_{R R}}^{2} \simeq(1-\rho) m_{0}^{2}+0.3 M_{1 / 2}^{2}
$$

with $\rho$ being a positive coefficient dependent on $\tan \beta$ and smaller than one in the present case, ${ }^{8}$ and

$$
\begin{aligned}
m_{\tilde{\tau}_{L R}}^{2} & =m_{\tau}\left(A_{\tau}-\mu \tan \beta\right) \\
& \simeq-m_{\tau} \mu \tan \beta
\end{aligned}
$$

where we explicitly indicate the dominance of the $\tan \beta$ enhanced term. In first approximation the lightest eigenvalue of eq. (4.3) is given by:

$$
\begin{aligned}
m_{\tilde{\tau}_{1}}^{2} & \simeq m_{\tilde{\tau}_{R R}}^{2}-m_{\tau} \mu \tan \beta \\
& \simeq(1-\rho) m_{0}^{2}+0.3 M_{1 / 2}^{2}-m_{\tau} \mu \tan \beta
\end{aligned}
$$

\footnotetext{
${ }^{8}$ On the contrary, when $\left|A_{0}\right| \neq 0$ the parameter $\rho$ could be driven larger than one by running effects due to the A-term. This could cause the arising of tachyonic stau masses, as we will discuss in section 4.4.
} 

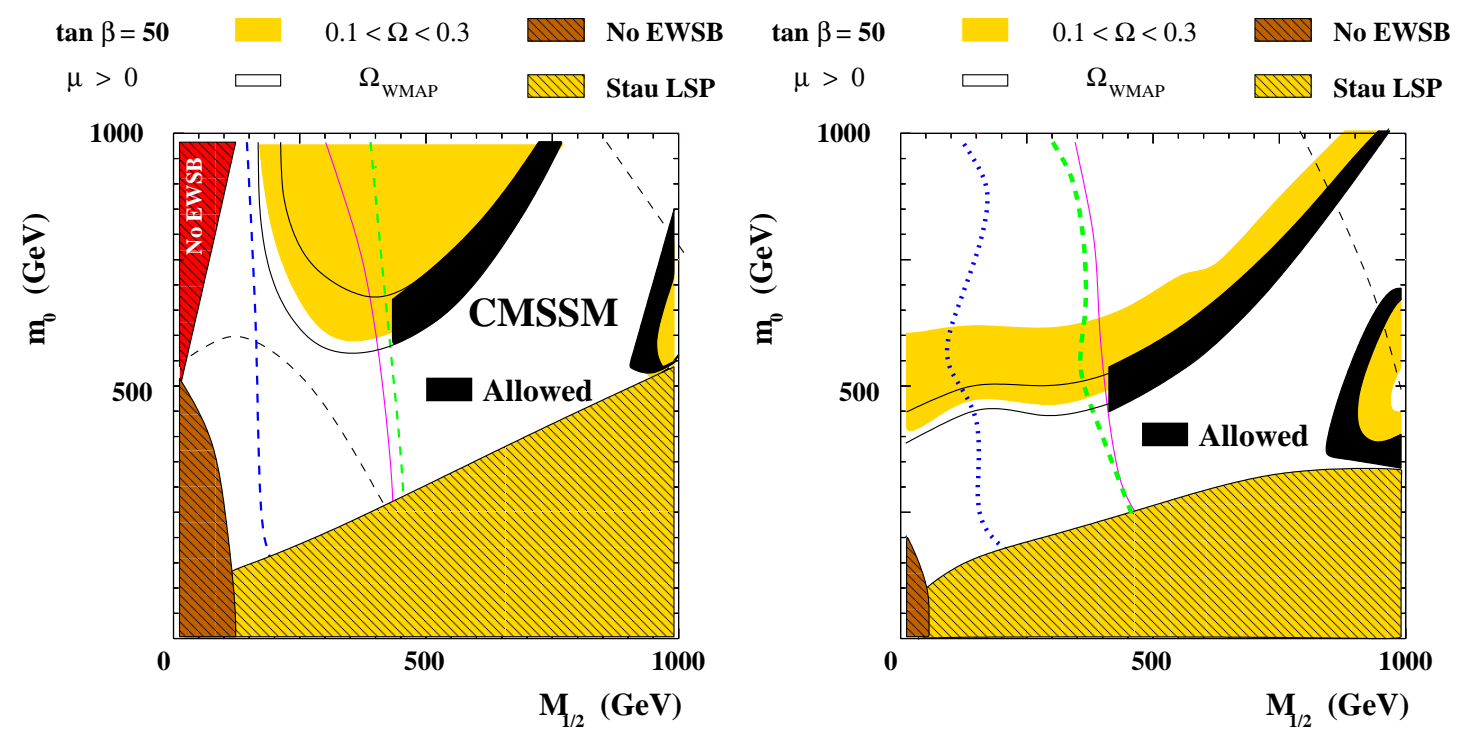

Figure 6: Same as above for $\tan \beta=50$.

The coannihilation condition requires that $m_{\tilde{\tau}_{1}}$ should be almost degenerate with the mass of the LSP, which is mostly the Bino mass, $M_{1} \simeq 0.47 M_{1 / 2}$. Taking $m_{0} \approx M_{1 / 2} \approx \mu \approx$ $M_{\mathrm{SUSY}}$ and barring the $\tan \beta$ dependence of the coefficient $\rho$, we find as a result $\tan \beta \simeq$ $(1-\rho)\left(M_{\mathrm{SUSY}} / m_{\tau}\right)$, from which, for reasonable values of $M_{\text {SUSY }} \gtrsim 200 \mathrm{GeV}$ and considering that in the present case $0.1 \lesssim \rho \lesssim 0.7$, we get the lower limit $\tan \beta \gtrsim 35 .{ }^{9}$ Solving numerically the system with the parameters varying in a wide region of the parameter space $\left(0 \leq m_{0}, M_{1 / 2} \leq 1 \mathrm{TeV}, 5 \leq \tan \beta \leq 50, A_{0}=0\right)$, we find that that there is no solution for $\tan \beta \lesssim 27$, furthermore, for a given $\tan \beta$, only a restricted region of the parameter space in $M_{1 / 2}$ is allowed. In figure 4 this region corresponds to the lower strip of points $34 \lesssim \tan \beta \lesssim 45$. As we can see, the limit on $\tan \beta$ is greater after applying the experimental constrains mainly due to restrictions coming from the bound on $m_{h}$. Overall, the spectrum prefers a neutralino mass of at least of $\mathcal{O}(150 \mathrm{GeV})$ and further with an upper bound of about $\mathcal{O}(200 \mathrm{GeV})$ if stau coannihilation is the dominant process in efficiently reducing the relic density to the observed levels. ${ }^{10}$

\subsection{A-pole funnel}

Let us now move to the funnel region. As in the CMSSM, this region makes its appearance for large $\tan \beta \simeq 45-50$. In figure 6, we have plotted the funnel region for $\tan \beta=$ $50, A_{0}=0$, again in comparison with the CMSSM case. We can also see large regions of the parameter space where the lightest stau is the LSP and thus there are regions of

\footnotetext{
${ }^{9}$ A longer running, as in the case $M_{X}=M_{\text {Planck, }}$, would increase $m_{\tilde{\tau}_{R R}}^{2}$ in eq. 4.6.). As a consequence an even larger value of $\tan \beta$ would be necessary to satisfy to coannihilation condition (though the larger $m_{\tilde{\tau}_{R R}}^{2}$ would be partially compensated by the simultaneous enhancing of $M_{1}$ ).

${ }^{10}$ Such a narrow range can perhaps be useful to distinguish this model compared to standard CMSSM at colliders.
} 
coannihilation also which are fused with the funnel region. The trunk region is no longer present and neither do the upper bounds.

\subsection{Focus point region}

As discussed in section 3, the REWSB is very easy to achieve due to the presence of additional GUT effects and top like Yukawa contribution from the neutrino Yukawa coupling to the up type Higgs between $M_{X}$ and $M_{R}$. The result is that there is no focus-point region present. We could not find the focus-point until $\left(m_{0}, M_{1 / 2}\right) \simeq 5 \mathrm{TeV}$. We have not explored further, since these regions would be far from the reach of LHC.

\section{$4.4\left|A_{0}\right|=3 m_{0}$}

All the solutions discussed above are for the special case where $A_{0}=0$ at the high scale. Given the constrained solutions we have here specifically for the stau coannihilation process, which can depend significantly on the non-zero $A_{0}$ parameter, it is instructive to consider the extreme cases of $A_{0}=-3 m_{0}$ and $A_{0}=+3 m_{0}$. For $A_{0}=-3 m_{0}$, we found that the occurrence of large regions of the $\left(m_{0}, M_{1 / 2}\right)$ plane excluded by tachyonic $m_{\tilde{\tau}_{1}}^{2}$ makes such case really disfavoured, leaving only a small portion of the parameter space viable. On the other hand, for $A_{0}=+3 m_{0}$ such excluded region results much smaller and localized in the portion of the plane where $m_{0}$ is large and $M_{1 / 2}$ small. Such behaviour can be explained by looking again at eq. (4.6). If the parameter $\rho$ there, which depends on $Y_{\tau}^{2}$ and $A_{\tau}^{2}$, becomes greater than one because the additional A-term running effect with respect to the case $A_{0}=0$, the coefficient in front of $m_{0}^{2}$ becomes negative. Therefore, values of $m_{0}^{2}$ larger than $M_{1 / 2}^{2}$ can easily make the lightest slepton tachyonic. The effect is enhanced by growing $\tan \beta$ both because the direct $\tan \beta$ dependence in eq. (4.6) and because the $\tan \beta$-enhanced $Y_{\tau}^{2}$ and $A_{\tau}^{2}$ contribution to the value of $\rho$. The different behaviours for $A_{0}= \pm 3 m_{0}$ are due to the renormalization group running of $A_{\tau}$ itself, which is not independent of the sign of $A_{0}$. In figure 7, we plot, for the $A_{0}=+3 m_{0}$ boundary condition, the regions which have the relic density consistent with WMAP measurements. We see from the left figure that $A_{0}=+3 m_{0}$ is very seriously constrained in the $\left(\tan \beta, M_{\chi_{1}^{0}}\right)$ plane compared to the $A_{0}=0$ case. The appearance of an upper bound $(\approx 45)$ on $\tan \beta$ is due to the increasing of the mentioned tachyonic- $\tilde{\tau}$ region for large values of $\tan \beta$, as it appears evident from eq. (4.6). Moreover, a closer look reveals a different branch of the coannihilation region in addition to the trunk region. This is shown in the right panel of figure 7 . We see in addition to the standard trunk branch, a thin strip on the top left corner, which appears along the $\tilde{\tau}$-LSP branch not present for $A_{0}=0$. Such branch runs along the large tachyonic- $\tilde{\tau}$ region which now appears due to the non-zero A-term as explained above.

\section{Remarks}

If supersymmetric standard models do really provide a dark matter candidate whose relic density constitutes most of the energy budget of the universe, one would typically expect non-trivial relations to hold true between various supersymmetric parameters. The point 

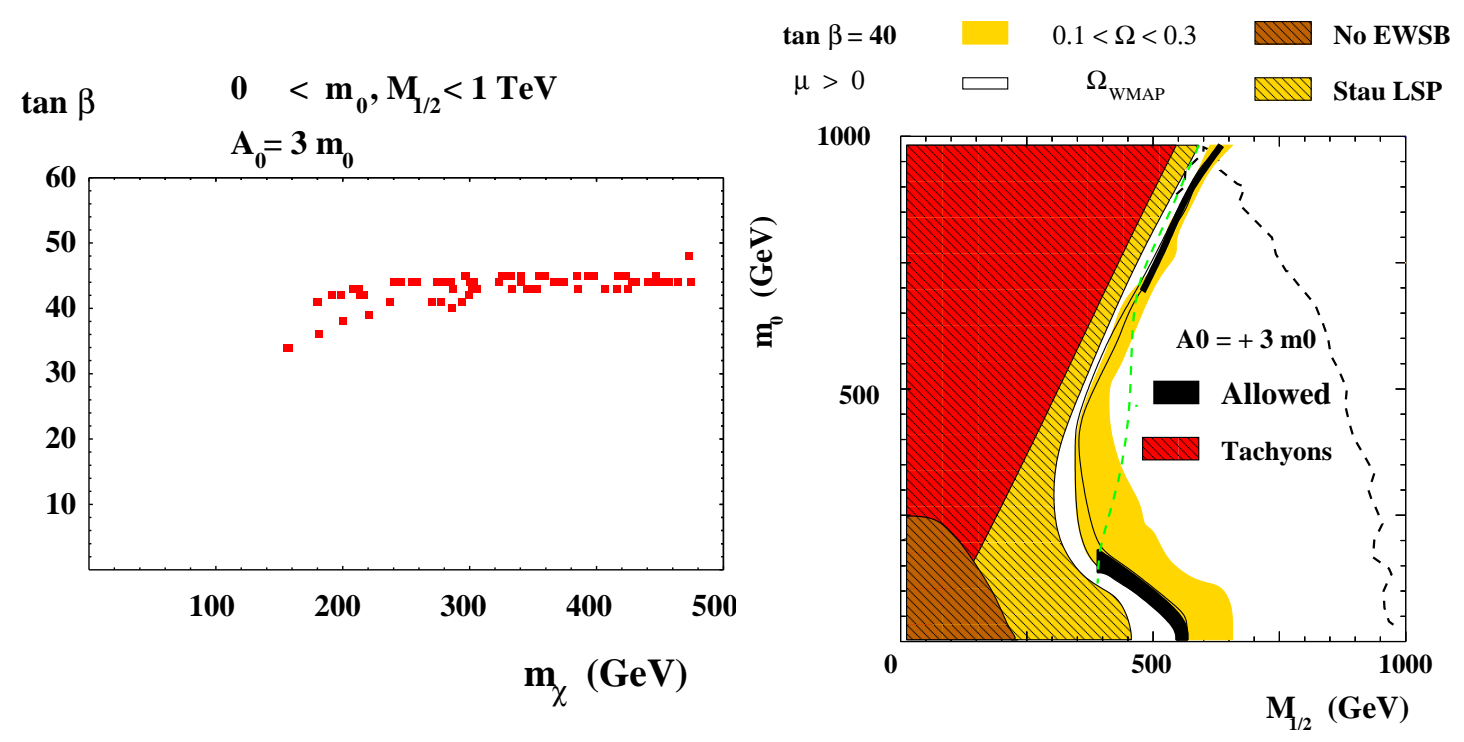

Figure 7: $A_{0}=3 m_{0}$ case. Left panel: points allowed by experimental and theoretical constrains after a scan on $\left(0<m_{0}, M_{1 / 2}<1 \mathrm{TeV}\right)$ and $(20<\tan \beta<55)$. Right panel: the $\left(m_{0}, M_{1 / 2}\right)$ plane plot for $\tan \beta=40$; we can see the two branches of the stau coannihilation region.

we tried to address was how stable these relations are under modifications to SUSY standard models. SUSY-GUTs with see-saw are natural extensions of MSSM in the light of experimental evidence for non-zero neutrino masses. In the simplest model we have considered here, $\mathrm{SU}(5)_{\mathrm{RN}}$, we found that relic density requirements put severe constraints on the range of $\tan \beta$. Only large $\tan \beta \gtrsim 35$ is now allowed. Such large $\tan \beta$ will also lead to large effects in flavour physics [24] which will also be present in this class of models.

Of the three possible corridors of viable parameter space present in CMSSM only two appear here. The stau coannihilation region is both bounded from above and below as long as $A_{0}=0$. The A-pole funnel appears for large $\tan \beta$, whereas focus-point region does not make an appearance in this class of models.

In the recent times, there has been a large interest in trying to reconstruct the dark matter relic density from SUSY spectrum measurements at colliders [28, 29]. While LHC alone might not be able to reconstruct the spectrum, one expects that astrophysical relic density measurements and LHC data would give a rough handle on the lightest neutralino mass. The couplings and cross-sections leading to precision measurements of the SUSY soft spectrum are possible at the ILC (International Linear Collider) operational in two modes with $\sqrt{s}=500 \mathrm{GeV}$ and $1 \mathrm{TeV}$. In particular, the use of $\tau$ polarization 30] should be able to distinguish this model from CMSSM as long as SUSY spectrum lies in the stau coannihilation region [31]. Here we use the fact that in our model, the relative sizes of $m_{\tilde{\tau}_{R R}}^{2}, m_{\tilde{\tau}_{L L}}^{2}$ and $m_{\tilde{\tau}_{L R}}^{2}$ change with respect to the CMSSM and consequently the large left-right mixing of stau required to get the degeneracy with the LSP. Further, it might be possible that the trunk region of this model, which has upper and lower bounds in the neutralino mass, can be tested from the data at LHC. It is not clear if the other regions which can have degenerate solutions with CMSSM can be discriminated either at LHC or 
a combination of LHC and future linear colliders. Such a question needs further studies.

Let us conclude by stressing that the peculiar phenomenology of the coannihilation we presented here turns out to be a quite general feature of GUT models. In fact, the crucial point is the increasing of the lightest stau mass due to effects of the unification gauge group such as in eq. 4.1). Similar effects are present every time $m_{\tilde{\tau}_{R}}^{2}$ feels the presence of a unified gauge sector, independently of particulars of the GUT structure and of the presence of right-handed neutrinos as in our case. Therefore, we claim that even in the absence of see-saw or large neutrino Yukawa couplings, the phenomenology of stau coannihilation regions would be similar in most GUT models. The presence of large see-saw couplings would effect the radiative electroweak symmetry breaking part and thus would have implications for the focus point regions. In the end our work shows that dark matter seems to predict large $\tan \beta$ in SUSY-GUTs.

\section{Acknowledgments}

We are highly grateful for discussions with D. P. Roy, X. Tata, R. Godbole and G. Polesello which have been highly informative and useful. We also acknowledge conversations with K. S. Babu, B. Ananthanarayan and P. Paradisi. SKV also acknowledges the organizers of the "From Strings to LHC" workshop at Goa, Jan, 2007, for the stimulating atmosphere where part of the work is done. The work of YM is sponsored by the PAI program PICASSO under contract PAI-10825VF. LC acknowledges the "Angelo Della Riccia" foundation, from which his work is supported, and the Theoretical Physics Department of the Valencia University for the kind hospitality and the stimulating environment.

\section{References}

[1] See. for a review, K. Griest and M. Kamionkowski, Supersymmetric dark matter, Phys. Rept. 333 (2000) 167.

[2] WMAP collaboration, C.L. Bennett et al., First year Wilkinson Microwave Anisotropy Probe (WMAP) observations: preliminary maps and basic results, Astrophys. J. Suppl. 148 (2003) 1 astro-ph/0302207;

WMAP collaboration, D.N. Spergel et al., First year Wilkinson Microwave Anisotropy Probe (WMAP) observations: determination of cosmological parameters, Astrophys. J. Suppl. 148 (2003) 175 astro-ph/0302209;

WMAP collaboration, L. Verde et al., First year Wilkinson Microwave Anisotropy Probe (WMAP) observations: parameter estimation methodology, Astrophys. J. Suppl. 148 (2003) 195 astro-ph/0302218.

[3] Particle Data Group collaboration, W.M. Yao et al., Review of particle physics, J. Phys. G $33(2006) 1$.

[4] For a recent discussion, please see, N. Arkani-Hamed, A. Delgado and G.F. Giudice, The well-tempered neutralino, Nucl. Phys. B 741 (2006) 108 hep-ph/0601041.

[5] U. Chattopadhyay, A. Corsetti and P. Nath, Wmap constraints, SUSY dark matter and implications for the direct detection of SUSY, Phys. Rev. D 68 (2003) 035005 hep-ph/0303201; 
H. Baer, C. Balázs, A. Belyaev, T. Krupovnickas and X. Tata, Updated reach of the CERN LHC and constraints from relic density, $b \rightarrow s \gamma$ and $a(m u)$ in the mSUGRA model, JHEP 06 (2003) 054 hep-ph/0304303;

J.R. Ellis, K.A. Olive, Y. Santoso and V.C. Spanos, Likelihood analysis of the cmssm parameter space, Phys. Rev. D 69 (2004) 095004 hep-ph/0310356.

[6] K. Griest and D. Seckel, Three exceptions in the calculation of relic abundances, Phys. Rev. D 43 (1991) 3191.

[7] J.R. Ellis, T. Falk and K.A. Olive, Neutralino stau coannihilation and the cosmological upper limit on the mass of the lightest supersymmetric particle, Phys. Lett. B 444 (1998) 367 hep-ph/9810360.

[8] J.R. Ellis, T. Falk, K.A. Olive and M. Srednicki, Calculations of neutralino stau coannihilation channels and the cosmologically relevant region of MSSM parameter space, Astropart. Phys. 13 (2000) 181 [Erratum ibid. 15 (2001) 413] hep-ph/9905481].

[9] M. Drees and M.M. Nojiri, The neutralino relic density in minimal $N=1$ supergravity, Phys. Rev. D 47 (1993) 376 hep-ph/9207234.

[10] K.L. Chan, U. Chattopadhyay and P. Nath, Naturalness, weak scale supersymmetry and the prospect for the observation of supersymmetry at the Tevatron and at the LHC, Phys. Rev. D 58 (1998) 096004 hep-ph/9710473;

J.L. Feng, K.T. Matchev and T. Moroi, Multi-TeV scalars are natural in minimal supergravity, Phys. Rev. Lett. 84 (2000) 2322 hep-ph/9908309;

J.L. Feng, K.T. Matchev and F. Wilczek, Neutralino dark matter in focus point supersymmetry, Phys. Lett. B 482 (2000) 388 hep-ph/0004043.

[11] A. Masiero, S.K. Vempati and O. Vives, Seesaw and lepton flavour violation in SUSY $\mathrm{SO}(10)$, Nucl. Phys. B 649 (2003) 189 hep-ph/0209303.

[12] Y. Mambrini and E. Nezri, Dark matter and colliders searches in the MSSM, Eur. Phys. J. C 50 (2007) 949 hep-ph/0507263;

H. Baer, T. Krupovnickas, S. Profumo and P. Ullio, Model independent approach to focus point supersymmetry: from dark matter to collider searches, JHEP 10 (2005) 020 hep-ph/0507282;

H. Baer, A. Mustafayev, E.-K. Park, S. Profumo and X. Tata, Mixed higgsino dark matter from a reduced $\mathrm{SU}(3)$ gaugino mass: consequences for dark matter and collider searches, JHEP 04 (2006) 041 hep-ph/0603197;

J.R. Ellis, T. Falk, K.A. Olive and Y. Santoso, Exploration of the MSSM with non-universal Higgs masses, Nucl. Phys. B 652 (2003) 259 hep-ph/0210205.

[13] S. Profumo, Neutralino relic density in supersymmetric guts with no-scale boundary conditions above the unification scale, JHEP 06 (2003) 052 hep-ph/0306119.

[14] L. Calibbi, A. Faccia, A. Masiero and S.K. Vempati, Lepton flavour violation from SUSY-GUTs: where do we stand for MEG, PRISM/PRIME and a super flavour factory, Phys. Rev. D 74 (2006) 116002 hep-ph/0605139.

[15] J. Hisano and D. Nomura, Solar and atmospheric neutrino oscillations and lepton flavor violation in supersymmetric models with the right-handed neutrinos, Phys. Rev. D 59 (1999) 116005 hep-ph/9810479. 
[16] A. Djouadi, J.-L. Kneur and G. Moultaka, SuSpect: a Fortran code for the supersymmetric and Higgs particle spectrum in the MSSM, Comput. Phys. Commun. 176 (2007) 426 hep-ph/0211331;

see also the web page http://www.lpta.univ-montp2.fr/users/kneur/Suspect/.

[17] A. Djouadi, M. Drees and J.L. Kneur, Constraints on the minimal supergravity model and prospects for SUSY particle production at future linear $e^{+} e^{-}$colliders, JHEP 08 (2001) 055 hep-ph/0107316.

[18] G. Bélanger, F. Boudjema, A. Pukhov and A. Semenov, MicrOMEGAs: a program for calculating the relic density in the MSSM, Comput. Phys. Commun. 149 (2002) 103 hep-ph/0112278; MicrOMEGAs: version 1.3, Comput. Phys. Commun. 174 (2006) 577 hep-ph/0405253; MicrOMEGAs2.0: a program to calculate the relic density of dark matter in a generic model, Comput. Phys. Commun. 176 (2007) 367 hep-ph/0607059.

[19] CLEO collaboration, S. Chen et al., Branching fraction and photon energy spectrum for $b \rightarrow s \gamma$, Phys. Rev. Lett. 87 (2001) 251807 hep-ex/0108032.

[20] BELLE collaboration, H. Tajima, Belle B physics results, Int. J. Mod. Phys. A 17 (2002) 2967 hep-ex/0111037.

[21] For a recent detailed analysis, please see: L. Roszkowski, R. Ruiz de Austri and R. Trotta, Implications for the constrained MSSM from a new prediction for $b \rightarrow s \gamma$, JHEP 07 (2007) 075 arXiv:0705.2012.

[22] M. Ciuchini et al., Soft SUSY breaking grand unification: leptons vs quarks on the flavor playground, hep-ph/0702144.

[23] K.-i. Okumura and L. Roszkowski, Weakened constraints from $b \rightarrow s \gamma$ on supersymmetry flavor mixing due to next-to-leading-order corrections, Phys. Rev. Lett. 92 (2004) 161801 hep-ph/0208101.

[24] A. Masiero, S.K. Vempati and O. Vives, Massive neutrinos and flavour violation, New J. Phys. 6 (2004) 202 hep-ph/0407325.

[25] Muon G-2 collaboration, G.W. Bennett et al., Measurement of the negative muon anomalous magnetic moment to 0.7-ppm, Phys. Rev. Lett. 92 (2004) 161802 hep-ex/0401008.

[26] M. Davier, S. Eidelman, A. Hocker and Z. Zhang, Updated estimate of the muon magnetic moment using revised results from $e^{+} e^{-}$annihilation, Eur. Phys. J. C 31 (2003) 503 hep-ph/0308213;

K. Hagiwara, A.D. Martin, D. Nomura and T. Teubner, Predictions for $g-2$ of the muon and $\alpha_{\mathrm{QED}}\left(M_{Z}^{2}\right)$, Phys. Rev. D 69 (2004) 093003 hep-ph/0312250;

J.F. de Troconiz and F.J. Yndurain, The hadronic contributions to the anomalous magnetic moment of the muon, Phys. Rev. D 71 (2005) 073008 hep-ph/0402285;

M. Passera, The standard model prediction of the muon anomalous magnetic moment, J Phys. G 31 (2005) R75 hep-ph/0411168.

[27] T. Nihei, L. Roszkowski and R. Ruiz de Austri, Exact cross sections for the neutralino slepton coannihilation, JHEP 07 (2002) 024 hep-ph/0206260;

J.R. Ellis, K.A. Olive, Y. Santoso and V.C. Spanos, Supersymmetric dark matter in light of WMAP, Phys. Lett. B 565 (2003) 176 hep-ph/0303043.

[28] E.A. Baltz, M. Battaglia, M.E. Peskin and T. Wizansky, Determination of dark matter properties at high-energy colliders, Phys. Rev. D 74 (2006) 103521 hep-ph/0602187. 
[29] M.M. Nojiri, G. Polesello and D.R. Tovey, Constraining dark matter in the MSSM at the LHC, JHEP 03 (2006) 063 hep-ph/0512204.

[30] M.M. Nojiri, Polarization of tau lepton from scalar tau decay as a probe of neutralino mixing, Phys. Rev. D 51 (1995) 6281 hep-ph/9412374;

R.M. Godbole, M. Guchait and D.P. Roy, Using tau polarization to discriminate between SUSY models and determine SUSY parameters at ILC, Phys. Lett. B 618 (2005) 193 hep-ph/0411306.

[31] L. Calibbi, R. Godbole, Y. Mambrini and S.K. Vempati, Distinguishing SUSY-GUT from mSUGRA at a linear collider, talk presented by R. Godboble at International Linear Collider Workshop 'LCWS 2007/ILC 2007', 30 May to 3 June 2007, Hamburg Germany, arXiv: to appear. 